\title{
Consensus-Based Approach for Perimeter Control of Urban Road Traffic Networks
}

\author{
Da-Biao Li, Zheng-Ran Liu, Wei Lang, Chao-Qun Zhang, Meng-Yao Zhu, Zhong-He He \\ Beijing Key Lab of Urban Intelligent Traffic Control Technology, \\ North China University of Technology, Beijing, China
}

\begin{abstract}
Continuous increasing of traffic demands often more than the network supply is the primary reason of traffic congestion. Recently, the existence of macroscopic fundamental diagram in urban traffic network has been validated and moreover activated the researches for the perimeter control of the network. Then, the centralized state-feedback design approach for the perimeter control of the network, with consensus-based control objective, is developed in this paper, which can realize the balance of network flows and moreover guarantee the maximum network capacity in saturated state of the network. A state-space model of the network perimeter control, with link occupancies as state variables and perimeter flows as control inputs, is first proposed. Furthermore, the consensus-based state-feedback control design approach is developed by using partial stability theory, and the consensus decision function of the closed-loop system can be analytically computed. Also, the distributed signal control approach of the network under the proposed perimeter control law is proposed, realizing the integration of the perimeter and signal control of the network.
\end{abstract}

Keywords-urban traffic control; perimeter control; consensus

\section{INTRODUCTION}

Traffic congestion frequently occurs in most of big cities, resulting in some serious problems, such as traffic pollution, accidents, and economic losses, etc, which has to be faced by the traffic management departments. At present, traffic signal control in the network is still an extensively adopted way for controlling dynamic traffic flows, though the technology of connected vehicles would provide the fundamental solution to traffic problems. Anyway, it has been proved that advanced control methods from control theory are feasible and effective for current urban infrastructure [1-2]. Then, large amounts of different modeling and optimization methods for urban traffic networks have been proposed, such as CTM-based generic algorithms [3-4], MPC-based approaches [5-6], optimal control approaches [7-9], hybrid system modeling and control approaches [10-14], Consensus-based approaches [15-19], etc. However, most of approaches are theoretical and lack field investigations. Also, from the views of practical applications, the SCOOT and SCATS systems, though extensively applied in many cities, mainly be suitable for non-saturated networks. Thus, the traffic control for the over-saturated networks is still a challenging problem.

The fundamental reason for the urban congestion is that traffic demands are more than network supply, resulting in capacity deficiency and then the oversaturation of the network. Recently, the network macroscopic fundamental diagram (NMFD), similar to the MFD for highway, is proved existence in urban networks [20-22]. Although, there are still deep investigations for properties of NMFD, the finding of NMFD has activated extensive researches for the perimeter control of the networks, especially for over-saturated networks.

For the problems of the perimeter control of the networks, the maximum accumulation of NMFD is typically chosen as the control objective, guaranteeing the maximum network production and then the maximum network capacity. For the single region, the proportional-integral controller was proposed in [23-24], where the region is seen as the basic unit with the accumulation of the network as state variable. For the multi-region coordination, the modeling ideas of the single region are extended by considering the coupling between multi-regions, e.g., LQR-based approach [25], MPC-based approach [26], and Robust control approach [27], etc.

In this paper, the problem of the perimeter control of the networks is investigated, and the consensus-based approach is proposed. Main contributions of the paper are as follows: 1. the multi-variable model for single region control is proposed with link occupancies as state variables, differently from single-variable model; 2 . the consensus-based approach is developed for the perimeter control, realizing the balance of link occupancies. Also, the consensus-state estimate for network link occupancies can be analytically computed; 3. a traffic-responsive signal control approach with the perimeter control law is presented.

\section{STATE-SPACE MODEL OF NETWORK CONTROL}

We represent by $J$ the set of signalized junctions in the network; by $V=\{1,2, \cdots\}$ the set of links in the network; by $V^{S}$ and $V^{D}$ respectively the set of input and output links on the network boundary; and by $V^{I}$ the set of internal links in the network.

From the basic conservation principle of network flows, the update law of traffic states in link $j \in V^{I}$ (see Figure 1) is determined by:

$$
N_{j}(t+1)=N_{j}(t)+Q_{j, \text { in }}(t)-Q_{j, \text { out }}(t)
$$

where $t \in \mathbb{N}$ is the discrete-time variable with $\mathbb{N}$ the set of natural number; $N_{j}(t)$ is the number of vehicles in link $j$ at the time $t$; both $Q_{j, \text { in }}(t)$ and $Q_{j, \text { out }}(t)$ are the total number of vehicles, respectively entering and leaving link $j$ in the sampled period $T$; and both $Q_{j \text {,in }}(t)$ and $Q_{j, \text { out }}(t)$ are respectively determined by: 


$$
\begin{gathered}
Q_{j, \text { in }}(t)=\sum_{i_{q} \in V_{j}^{J}} \alpha_{i_{q} j}\left[\eta_{i_{q}}(t) N_{i_{q}}(t)\right]+\sum_{i_{q} \in E j} \alpha_{i_{q}} Q_{i_{q}}(t), \\
Q_{j, \text { out }}(t)=\sum_{k_{q} \in V_{j}^{D}} \alpha_{j k_{q}}\left[\eta_{j}(t) N_{j}(t)\right],
\end{gathered}
$$

where $V_{j}^{S}, V_{j}^{I}$, and $V_{j}^{D}$ are respectively the set of upstream input links, of upstream internal links, and of downstream links of link $j ; Q_{i}(t), i \in V^{S}$ is the number of vehicles entering the network from input link $i$ in the sampled period $T ; \alpha_{i j} \geq 0, \quad i \in V^{S} \cup V^{I}$, assumed to be constant or piecewise-constant determined from real data in practical applications, is the distributing proportion of traffic flows from link $i$ to downstream link $j$, and satisfies: $\sum_{j \in V_{i}^{\mathrm{D}}} \alpha_{i j}=1$; and $\eta_{i}(t) \triangleq Q_{i, \text { out }}(t) / N_{i}(t), \quad i \in V^{I}$ is the discharging proportion of traffic flows in link $i$ in the sampled period $T$, which quantitatively evaluates saturation levels in the link, e.g., $\eta_{i}(t)<1$ indicates that the queue in the link does not dissipate in one cycle.

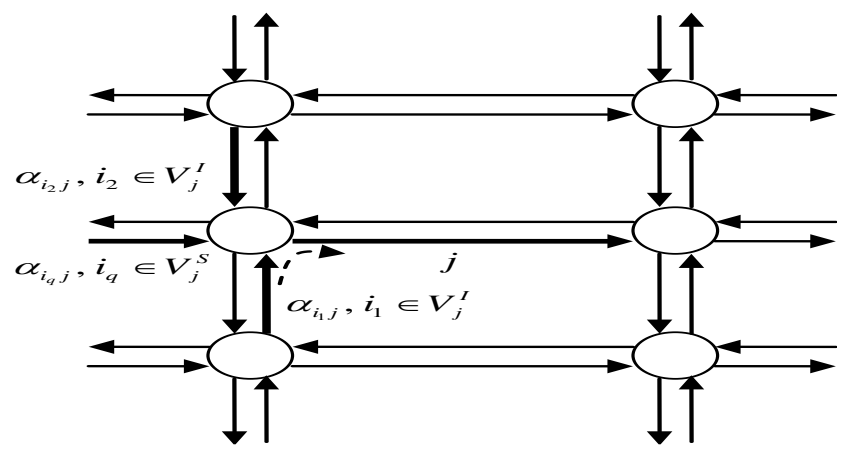

Figure 1. Dynamics of the link.

Furthermore, assume that the following two terms hold.

Assumption 1. The sampled period of the model in (1) equals to the common cycle $C$ of the network, i.e., $T=C$.

Assumption 2. The links $i \in V^{S} \cup V^{I}$ discharge the queues at the saturation flows.

Remark 1. For the coordination among signalized junctions in the network, the common cycle is typically applied for traffic control of the network. The model in (1) under Assumption 1 basically follows the store-and-forward modeling approaches, originally suggested in [28], and then successfully applied in [6-8], the implication of which is that the model in (1) describes the dynamics of traffic flows in links each cycle, ignoring fluctuations of traffic flows within the cycle, and then eliminating the influence of the red-green signal switching and reducing the complexity of modeling and optimization of the network. Then, the model in (1) can be seen as a traffic control design model, not a simulation model. The common cycle $C$ of the network may be obtained from other parallel off-line or on-line optimization approaches, and we focus on the designs of splits and network inputs under the given cycle and offsets of the network.

Substituting (2) and (3) to (1), the state-space model of network control is obtained by:

$$
N(t+1)=A(t) N(t)+B Q(t)
$$

where $N(t)=\left[N_{1}(t), \ldots, N_{n}(t)\right]^{T} \in \mathbb{R}^{n}$ is the state vector $\left(\operatorname{dim}(N)=\left|V^{I}\right|=n\right.$ with $|\cdot|$ denoting the cardinality of the set); $A(t)=\left[a_{i j}(t)\right] \in \mathbb{R}^{n \times n}$ is the state matrix with diagonal elements $a_{i i}(t)=1-\eta_{i}(t), i=1, \ldots, n$, and others

$$
a_{i j}(t)=\left\{\begin{array}{ll}
\alpha_{j i} \eta_{j}(t), & j \in V_{i}^{I} \\
0, & \text { otherwise }
\end{array} ;\right.
$$

$Q(t)=\left[Q_{1}(t), \ldots, Q_{m}(t)\right]^{T} \in \mathbb{R}^{m}$ is the control input vector of the network, i.e., traffic demands of the network $\left(\operatorname{dim}(Q)=\left|V^{S}\right|=m\right)$; and $B=\left[b_{i j}\right] \in \mathbb{R}^{n \times m}$ is the input matrix with elements

$$
b_{i j}=\left\{\begin{array}{ll}
\alpha_{j i}, & j \in V_{i}^{S} \\
0, & \text { otherwise }
\end{array} .\right.
$$
form:

Furthermore, introduce the linear transformation of the

$$
x=L N \text { with } L=\operatorname{diag}\left\{1 / N_{1, \max }, \ldots, 1 / N_{n, \text { max }}\right\}
$$

where $x=\left[x_{1}, \ldots, x_{n}\right]^{T} \in \mathbb{R}^{n}$, and $L$ is a diagonal matrix with $N_{i, \max }$ being the maximum number of vehicles admitted in link $i$.

Apply (5) to (4), we have that

$$
x(t+1)=\left[L A(t) L^{-1}\right] x(t)+L B Q(t),
$$

where $x(t)=\left[x_{1}(t), \ldots, x_{n}(t)\right]^{T}$ with $x_{i}(t)=N_{i}(t) / N_{i, \max }$; $L^{-1}$ is the inverse matrix of $L$.

The state variable $x_{i}(t)=N_{i}(t) / N_{i, \max }$ in (6) is called the relative occupancy of vehicles in link $i$ (called link occupancy for short), which can reflect congestion levels in the link. In the following Section, a state-feedback control law is designed for (6), under which state components of the closed-loop system can asymptotically reach consensus, implying that to some extent the distribution of traffic flows in network links is balanced, which can reduce local congestion levels and then the risk of link overflows.

\section{FEEDBACK CONTROL DESIGN OF NETWORK INPUTS}

In this Section, we first introduce some results about the M-matrix, and then develop the consensus-based feedback control design approaches by using partial stability theory.

\section{A. Preliminary Results about M-matrix}

We first introduce some definitions and properties for the special class of matrices (refer to [29] for details). In the following parts, the symbol " $P>0$ " represents that all the elements in the matrix (or vector) $P$ are greater than or equal to zero and $P \neq 0$; and the symbol " $P>>0$ " represents that 
all the elements in the matrix (or vector) $P$ are greater than zero. The matrix $P=\left[p_{i j}\right] \in \mathbb{R}^{n \times n}$ is called an M-matrix, if $p_{i j} \leq 0, i, j=1, \ldots, n, i \neq j$ and real parts $\operatorname{Re} \lambda \geq 0$ for all $\lambda \in \operatorname{spec}(P)$, the spectrum of $P$. If the matrix $P$ is a nonsingular M-matrix, then $P^{-1}>0$.

Lemma 1 [18]. For the any given $\bar{\eta}=\left[\bar{\eta}_{1}, \ldots, \bar{\eta}_{n}\right]^{T}>>$, $\tilde{A} \triangleq E_{n}-\bar{A} \in \mathbb{R}^{n \times n}$ is a nonsingular M-matrix, where $E_{n}$ is the $n$-dimensional unit matrix and $\bar{A}=\left.A(t)\right|_{\eta_{i}(t)=\bar{\eta}_{i}, i=1, \ldots, n}$ with $A(t)$ defined in (4).

It is derived from Lemma 1 that for the any given $\bar{Q}=\left[\bar{Q}_{1}, \ldots, \bar{Q}_{m}\right]^{T}>>$ and $\bar{\eta}=\left[\bar{\eta}_{1}, \ldots, \bar{\eta}_{n}\right]^{T}>>$, there exists a unique equilibrium $\bar{N}=\left[\bar{N}_{1}, \ldots, \bar{N}_{n}\right]^{T}$ for (4) with $Q(t) \equiv \bar{Q}$ and $A(t) \equiv \bar{A}$, i.e., $\bar{N}=\tilde{A}^{-1} B \bar{Q}$.

Proposition 1. Define the matrix $\tilde{A}_{1} \triangleq E_{n}-\bar{A}_{1} \in \mathbb{R}^{n \times n}$, where $\bar{A}_{1}=\left.A(t)\right|_{\eta_{i}(t)=1, i=1, \ldots, n}$ and $E_{n}$ is the $n$-dimensional unit matrix. Then, for any given scalar $a<1$, there exists a unique solution $\bar{\eta}=\left[\bar{\eta}_{1}, \ldots, \bar{\eta}_{n}\right]^{T}>>0$ to the linear system of equations $\left[L \tilde{A}_{1} L^{-1}\right] \bar{\eta}=(1-a) 1_{n}$, where $L$ is defined in (5), and $1_{n} \in \mathbb{R}^{n}$ with all elements equal to one.

Proof: From Lemma 1, the defined matrix $\tilde{A}_{1}=E_{n}-\bar{A}_{1}$ is a nonsingular M-matrix, and then $L \tilde{A}_{1} L^{-1}$ is also a nonsingular M-matrix implying $\left[L \tilde{A}_{1} L^{-1}\right]^{-1}>0$. Thus, there exists a unique solution to $\left[L \tilde{A}_{1} L^{-1}\right] \bar{\eta}=(1-a) 1_{n}$ for the any given scalar $a \in \mathbb{R}$. Furthermore, from $a<1$ and $\left[L \tilde{A}_{1} L^{-1}\right]^{-1}>0$, we have $\bar{\eta}=(1-a)\left[L \tilde{A}_{1} L^{-1}\right]^{-1} 1_{n}>>0$.

The elements of $\left[L \tilde{A}_{1} L^{-1}\right]^{-1}$ depend on link capacities and distributing proportions of traffic flows in the network, and discharging proportions $\bar{\eta}>>0$ of links can be adjusted by choosing different $a$ and monotonously decrease with $a$. For some chosen $a<1$, the solution to $\left[L \tilde{A}_{1} L^{-1}\right] \bar{\eta}=(1-a) 1_{n}$ is noted as $\bar{\eta}^{a}=\left[\bar{\eta}_{1}^{a}, \ldots, \bar{\eta}_{n}^{a}\right]^{T}$, where the superscript represents the relevance to the chosen $a$. It is derived from Proposition 1 that the sum of each of rows of the matrix $L \bar{A}^{a} L^{-1}$ with $\bar{A}^{a}=\left.A(t)\right|_{\eta_{i}(t)=\eta_{i}^{a}, i=1, \ldots, n}$, is equal to $a$, i.e., $\left[L \bar{A}^{a} L^{-1}\right] 1_{n}=a 1_{n}$.

Now, consider (6) with $\bar{\eta}^{a}=\left[\bar{\eta}_{1}^{a}, \ldots, \bar{\eta}_{n}^{a}\right]^{T}$ as discharging proportions of internal links, then

$$
x(t+1)=\left[L \bar{A}^{a} L^{-1}\right] x(t)+L B Q(t)
$$

The objective of the following parts is to design a state-feedback control law, under which (7) can reach consensus.

\section{B. General Results of Consensus of Linear Systems}

Consider the following discrete-time linear time-invariant system:

$$
x(t+1)=A x(t), \quad x(0)=x_{0}, t \in \mathbb{N},
$$

where $x(t)=\left[x_{1}(t), \ldots, x_{n}(t)\right]^{T} \in \mathbb{R}^{n}$, and $A \in \mathbb{R}^{n \times n}$ is the state matrix.

Let $\quad y(t)=\left[x_{1}(t), \ldots, x_{n-1}(t)\right]^{T} \quad, \quad$ and $y_{0}=\left[x_{1}(0), \ldots, x_{n-1}(0)\right]^{T}$.

Definition 1. (Partial Stability (see Figure 2 for illustration)) The equilibrium $x=0$ of (8) is stable with respect to partial variables $y$ ( $y$-stable for short), if for $\forall \varepsilon>0, \exists \delta(\varepsilon)>0$, such that $\left\|y_{0}\right\|<\delta(\|\cdot\|$ denotes some type of norm) implies $\|y(t)\|<\varepsilon, \forall t \in \mathbb{N}$. Furthermore, the equilibrium $x=0$ of (8) is asymptotically stable with respect to partial variables $y$ (asymptotic $y$-stable for short), if (8) is $y$-stable and partial variables $y$ satisfies $\lim _{t \rightarrow+\infty}\|y(t)\|=0$.

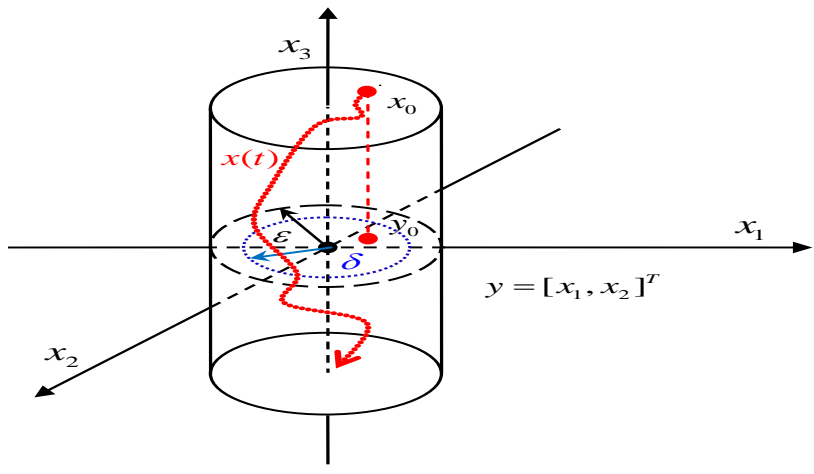

Figure 2. Illustration for partial stability.

Definition 2. (Asymptotic Stable Consensus) If there exists a scalar function $x^{*}(t) \in \mathbb{R}, t \in \mathbb{N}$ with $\lim _{t \rightarrow+\infty}\left[x_{i}(t)-x^{*}(t)\right]=0, \quad \forall i \in\{1, \ldots, n\}$, then (8) is called to realize asymptotic consensus, and $x^{*}(t)$ is called the consensus decision function. Furthermore, if for $\forall \varepsilon>0$, $\exists \delta(\varepsilon)>0$, such that $\left\|x_{0}-x^{*}(0) 1_{n}\right\|<\delta$ implies $\left\|x(t)-x^{*}(t) 1_{n}\right\|<\varepsilon, \forall t \in \mathbb{N}$, then the consensus decision function $x^{*}(t)$ is called to be stable. If (8) can realize asymptotic consensus and the consensus decision function $x^{*}(t)$ is stable, (8) is called to realize asymptotic stable consensus.

Construct the transformation matrix $P \in \mathbb{R}^{n \times n}$ to (8) as follows: 


$$
P=\left[\begin{array}{c}
p_{1} \\
\vdots \\
p_{n-1} \\
1_{n}^{T}
\end{array}\right]=\left[\begin{array}{c}
P_{1} \\
1_{n}^{T}
\end{array}\right],
$$

where $\quad p_{i}^{T}=\left[p_{i, 1}, \ldots, p_{i, n}\right]^{T} \in \mathbb{R}^{n}, i=1, \ldots, n-1 \quad$ are linearly independent and satisfy $p_{i} 1_{n}=0$, and $P_{1} \in \mathbb{R}^{(n-1) \times n}$ is the first $n-1$ rows of matrix $P$.

Proposition 2. The inverse $P^{-1} \triangleq \bar{P}$ of matrix $P$ has the following form:

$$
\bar{P}=\left[\begin{array}{llll}
\bar{p}_{1} & \cdots & \bar{p}_{n-1} & n^{-1} 1_{n}
\end{array}\right]=\left[\begin{array}{ll}
\bar{P}_{1} & n^{-1} 1_{n}
\end{array}\right],
$$

where $\bar{P}_{1} \in \mathbb{R}^{n \times(n-1)}$ is the first $n-1$ columns of matrix $\bar{P}$ and $\bar{p}_{i}=\left[\bar{p}_{1, i}, \ldots, \bar{p}_{n, i}\right]^{T} \in \mathbb{R}^{n}, i=1, \ldots, n-1$.

Remark 2. Since $P \bar{P}=E_{n}$, where $E_{n} \in \mathbb{R}^{n \times n}$ is the unit matrix, we have $P_{1} \bar{P}_{1}=E_{n-1}$ and $1_{n}^{T} \bar{P}_{1}=0$. Moreover, from $\bar{P} P=E_{n}$, we have $\bar{P}_{1} P_{1}=E_{n}-n^{-1} 1_{n} 1_{n}^{T}$.

Apply the linear transformation $\bar{x}=P x$ to (8), we have

$$
\bar{x}(t+1)=P A P^{-1} \bar{x}(t)
$$

where $\bar{x}(t)=\left[\bar{x}_{1}(t), \ldots, \bar{x}_{n}(t)\right]^{T} \in \mathbb{R}^{n}$.

Theorem 1. (8) can realize asymptotic stable consensus if and only if the equilibrium $\bar{x}=0$ of (11) is asymptotically stable with respect to partial variables $y=\left[\bar{x}_{1}, \ldots, \bar{x}_{n-1}\right]^{T}$.

Remark 3. The proofs of Proposition 2 and Theorem 1 can be referred to [15]. The results in Theorem 1 are of importance, which establish the equivalent relation between the consensus of the original system and the partial stability of the transformed system. From $\bar{x}=P x$, we have $x=P^{-1} \bar{X}=\bar{P} \bar{X} \quad$ with $\bar{P} \quad$ defined in (10), then $x_{i}=\sum_{j=1}^{n-1} \bar{p}_{i j} \bar{x}_{j}+n^{-1} \bar{x}_{n}, \quad \forall i \in\{1, \ldots, n\}$. Thus, from the results in Theorem 1 , the sufficient part indicates $\bar{x}_{j} \rightarrow 0, \forall j \in\{1, \ldots, n-1\} \quad, \quad$ and then $x_{i} \rightarrow n^{-1} \bar{x}_{n}$, $\forall i \in\{1, \ldots, n\}$, implying the consensus decision function of (8) is $x^{*}(t)=n^{-1} \bar{x}_{n}(t)$.

\section{Consensus-based Feedback Control Design}

The state-feedback control law for (7) is of the form:

$$
Q(t)=K R x(t)
$$

where $K \in \mathbb{R}^{m \times(n-1)}$ is the solved feedback-gain matrix, and $R \in \mathbb{R}^{(n-1) \times n}$ is a known matrix satisfying technical condition $R 1_{n}=0$, e.g., $R=P_{1}$, where $P_{1}$ is defined in (9).

Substituting (12) in (7), closed-loop system is given by:

$$
x(t+1)=\left\{\left[L \bar{A}^{a} L^{-1}\right]+L B K R\right\} x(t) .
$$
that

Apply the linear transformation $\bar{x}=P x$ to (13), we have

$$
\bar{x}(t+1)=P\left\{\left[L \bar{A}^{a} L^{-1}\right]+L B K R\right\} P^{-1} \bar{x}(t),
$$

where $P$ and $P^{-1}$ are defined in (9) and (10), respectively.

It is derived from the properties of $P$ and $P^{-1}$, that the state matrix $P\left\{\left[L \bar{A}^{a} L^{-1}\right]+L B K R\right\} P^{-1}$ in (14) satisfies:

$$
\begin{aligned}
& P\left\{\left[L \bar{A}^{a} L^{-1}\right]+L B K R\right\} P^{-1} \\
= & {\left[\begin{array}{c}
P_{1} \\
1_{n}^{T}
\end{array}\right]\left\{\left[L \bar{A}^{a} L^{-1}\right]+L B K R\right\}\left[\begin{array}{ll}
\bar{P}_{1} & n^{-1} 1_{n}
\end{array}\right] } \\
= & {\left[\begin{array}{ll}
P_{1}\left\{\left[L \bar{A}^{a} L^{-1}\right]+L B K R\right\} \bar{P}_{1} & n^{-1} P_{1}\left\{\left[L \bar{A}^{a} L^{-1}\right]+L B K R\right\} 1_{n} \\
1_{n}^{T}\left\{\left[L \bar{A}^{a} L^{-1}\right]+L B K R\right\} \bar{P}_{1} & n^{-1} 1_{n}^{T}\left\{\left[L \bar{A}^{a} L^{-1}\right]+L B K R\right\} 1_{n}
\end{array}\right] } \\
= & {\left[\begin{array}{ll}
P_{1}\left\{\left[L \bar{A}^{a} L^{-1}\right]+L B K R\right\} \bar{P}_{1} & 0 \\
1_{n}^{T}\left\{\left[L \bar{A}^{a} L^{-1}\right]+L B K R\right\} \bar{P}_{1} & a
\end{array}\right] . }
\end{aligned}
$$

where $\quad\left[L \bar{A}^{a} L^{-1}\right] 1_{n}=a 1_{n} \quad$ and $\quad R 1_{n}=0 \quad$ respectively indicate

$$
\begin{aligned}
& n^{-1} P_{1}\left\{\left[L \bar{A}^{a} L^{-1}\right]+L B K R\right\} 1_{n}=0, \\
& n^{-1} 1_{n}^{T}\left\{\left[L \bar{A}^{a} L^{-1}\right]+L B K R\right\} 1_{n}=a .
\end{aligned}
$$

Let $\quad \bar{X}=\left[\bar{x}_{1}, \ldots, \bar{x}_{n-1}, \bar{x}_{n}\right]^{T}=\left[y^{T}, \bar{x}_{n}\right]^{T}$ with $y=\left[\bar{x}_{1}, \ldots, \bar{x}_{n-1}\right]^{T}$, then from (15), (14) can be represented as the block form:

$$
\left\{\begin{array}{l}
y(t+1)=\hat{A} y(t)+\hat{b} \bar{x}_{n}(t) \\
\bar{x}_{n}(t+1)=\hat{c} y(t)+\hat{d} \bar{x}_{n}(t)
\end{array}\right.
$$

where $\quad \hat{A}=P_{1}\left\{\left[L \bar{A}^{a} L^{-1}\right]+L B K R\right\} \bar{P}_{1} \in \mathbb{R}^{(n-1) \times(n-1)}$, $\hat{d}=a \in \mathbb{R} \quad \hat{b}=0 \in \mathbb{R}^{n-1} \quad, \quad$ and $\hat{c}=1_{n}^{T}\left\{\left[L \bar{A}^{a} L^{-1}\right]+L B K R\right\} \bar{P}_{1} \in \mathbb{R}^{1 \times(n-1)}$.

Theorem 2. The equilibrium $\bar{x}=0$ of (16) is asymptotically stable with respect to partial variables $y=\left[\bar{x}_{1}, \ldots, \bar{x}_{n-1}\right]^{T}$ if and only if the matrix $\hat{A}$ in (16) is Schur stable, i.e., the eigenvalues of $\hat{A}$ lie inside the interior of the unit circle.

Proof: Since $\hat{b}=0$ in (16), the evolution of partial variables $y=\left[\bar{x}_{1}, \ldots, \bar{x}_{n-1}\right]^{T}$ in (16) does not depend on $\bar{x}_{n}$. Then, the results in Theorem 2 can be derived from Definition 1.

Theorem 3. (13) can realize asymptotic stable consensus if and only if the matrix $\hat{A}$ in (16) is Schur stable.

Proof: The results in Theorem 3 can be directly derived from the results in Theorem 1 and Theorem 2 . 
Then, the results in Theorem 3 indicate that the problem of solving the feedback-gain matrix $K$ such that (13) can realize asymptotic stable consensus, is transformed to the problem of solving the matrix $K$ such that the matrix $\hat{A}$ in (16) is Schur stable, which is the standard problem of the feedback stabilization of linear systems. Then, the linear matrix inequality (LMI) method [30] can be used to numerically solve the feedback-gain matrix $K$.

If the closed-loop system (13) can realize asymptotic stable consensus, then the consensus decision function $x^{*}(t)$ of (13) can be analytically computed.

Proposition 3. Assume that the closed-loop system (13) can realize asymptotic stable consensus, i.e., from Theorem 3 the matrix $\hat{A}$ in (16) is Schur stable. Then, the consensus decision function $x^{*}(t)$ of (13) is obtained by:

$$
x^{*}(t)=n^{-1}\left[\hat{c}\left(\sum_{k=0}^{t-1} a^{k} \hat{A}^{t-1-k}\right) P_{1}+a^{t} 1_{n}^{T}\right] x(0), \quad \forall t \geq 1
$$

where $\hat{A}$ and $\hat{c}$ are respectively given in (16), and $P_{1}$ is defined in (9). Moreover, the coefficient in (17) satisfies:

$$
\begin{gathered}
n^{-1}\left[\hat{c}\left(\sum_{k=0}^{t-1} a^{k} \hat{A}^{t-1-k}\right) P_{1}+a^{t} 1_{n}^{T}\right]\left[L \bar{A}^{a} L^{-1}+L B K R\right] \\
=n^{-1}\left[\hat{c}\left(\sum_{k=0}^{t} a^{k} \hat{A}^{t-k}\right) P_{1}+a^{t+1} 1_{n}^{T}\right], \\
n^{-1}\left[\hat{c}\left(\sum_{k=0}^{t-1} a^{k} \hat{A}^{t-1-k}\right) P_{1}+a^{t} 1_{n}^{T}\right] 1_{n}=a^{t} .
\end{gathered}
$$

Proof: From the statements in Remark 3, we have that $x^{*}(t)=n^{-1} \bar{x}_{n}(t)$, where $\bar{x}_{n}(t)$ is the $n$th state variable in (16). Since $\hat{b}=0$ and $\hat{d}=a$ in (16), the solution to (16) with the initial state $\left[y^{T}(0), \bar{x}_{n}(0)\right]^{T}$ is given by:

$$
\left\{\begin{array}{l}
y(t)=\hat{A}^{t} y(0), \\
\bar{x}_{n}(t)=\hat{c}\left(\sum_{k=0}^{t-1} a^{k} \hat{A}^{t-1-k}\right) y(0)+a^{t} \bar{x}_{n}(0),
\end{array} \forall t \geq 1\right.
$$

Since $\bar{x}=P x$, we have $y(0)=P_{1} x(0)$ and $\bar{X}_{n}(0)=1_{n}^{T} x(0)$. Then, (17) can be derived by substituting $y(0)=P_{1} x(0)$ and $\bar{x}_{n}(0)=1_{n}^{T} x(0)$ into (20). Furthermore, let $W=L \bar{A}^{a} L^{-1}+L B K R$, i.e., the state matrix in (13), and from Remark 2, we have $W \bar{P}_{1} P_{1}=W\left[E_{n}-n^{-1} 1_{n} 1_{n}^{T}\right]=W-n^{-1} a 1_{n} 1_{n}^{T}$, then,

$$
\begin{aligned}
& n^{-1}\left[\hat{c}\left(\sum_{k=0}^{t-1} a^{k} \hat{A}^{t-1-k}\right) P_{1}+a^{t} 1_{1}^{T}\right] W \\
= & n^{-1}\left[\hat{c}\left(\sum_{k=0}^{t-1} a^{k} \hat{A}^{t-1-k}\right) P_{1}\left(W \bar{P}_{1} P_{1}+n^{-1} a 1_{n} n_{n}^{T}\right)+a^{t} 1_{n}^{T}\left(W \bar{P}_{1} P_{1}+n^{-1} a_{n} n_{n}^{T}\right)\right] \\
= & n^{-1}\left[\hat{c}\left(\sum_{k=0}^{t-1} a^{k} \hat{A}^{t-1-k}\right)\left(P_{1} W \bar{P}_{1}\right) P_{1}+a^{t}\left(1_{n}^{T} W \overline{P_{1}}\right) P_{1}+a^{t+1} 1_{n}^{T}\right] \\
= & n^{-1}\left[\hat{c}\left(\sum_{k=0}^{t-1} a^{k} \hat{A}^{t-1-k}\right) \hat{A} P_{1}+a^{t} \hat{c} P_{1}+a^{t+1} 1_{n}^{T}\right] \\
= & n^{-1}\left\{\hat{c}\left[\left(\sum_{k=0}^{t-1} a^{k} \hat{A}^{t-1-k}\right) \hat{A}+a^{t} E_{n-1}\right] P_{1}+a^{t+1} 1_{n}^{T}\right\} \\
= & n^{-1}\left[\hat{c}\left(\sum_{k=0}^{t} a^{a} \hat{A}^{t-k}\right) P_{1}+a^{t+1} 1_{n}^{T}\right],
\end{aligned}
$$

where $\hat{A}=P_{1} W \bar{P}_{1}$ and $\hat{c}=1_{n}^{T} W \bar{P}_{1}$. Then (18) is directly derived from (21). Furthermore, $P_{1} 1_{n}=0$ and $1_{n}^{T} 1_{n}=n$ indicate that $n^{-1}\left[\hat{c}\left(\sum_{k=0}^{t-1} a^{k} \hat{A}^{t-1-k}\right) P_{1}+a^{t} 1_{n}^{T}\right] 1_{n}=a^{t}$, i.e., (19) holds.

From Proposition 3, the evolution of the consensus decision function $x^{*}(t)$ in (17) is the weighed sum of components of the initial state $x(0)$, and then provides the consensus estimate for link occupancies of the network. Furthermore, from the closed-loop system (13) and (18), we have that $x^{*}(t)=n^{-1}\left[\hat{c} P_{1}+a 1_{n}^{T}\right] x(t-1)$, combined with $\lim _{t \rightarrow \infty}\left[x(t)-x^{*}(t) 1_{n}\right]=0$ and $P_{1} 1_{n}=0$, indicating that if the consensus decision function $x^{*}(t)$ converges to a finite value $\bar{X}^{*}$, then $\bar{x}^{*}=0$. Moreover (19) indicates the sum of weights equals to $a^{t}$ at the time $t$ and $\lim _{t \rightarrow \infty} a^{t}=0$ with $|a|<1$.

So far, we have provided the design approaches of the state-feedback control law for (7) realizing asymptotic stable consensus. Furthermore, assume that $Q^{e}=\left[Q_{1}^{e}, \ldots, Q_{m}^{e}\right]^{T}>>0$ is the expected inputs of the network, and then the equilibrium $x_{e}$ of (7) with $Q(t) \equiv Q^{e}>>0$ is given by:

$$
x_{e}=\left[L\left(E_{n}-\bar{A}^{a}\right) L^{-1}\right]^{-1} L B Q^{e}=L\left(E_{n}-\bar{A}^{a}\right)^{-1} B Q^{e} .
$$

Then, the applied state-feedback control law of the network inputs is given by $Q(t)=Q^{e}+K R\left(x(t)-x_{e}\right)$, where $Q(t)=\left[Q_{1}(t), \ldots, Q_{m}(t)\right]^{T}$ is the applied network input; $x(t)=\left[x_{1}(t), \ldots, x_{n}(t)\right]^{T}$ is the detected link occupancies of the network; and $K \in \mathbb{R}^{m \times(n-1)}$ is the feedback-gain matrix solved by above proposed approaches. However, the network inputs have to be constrained by the link capacity, then, practical inputs are eventually determined by:

$$
\tilde{Q}_{i}(t)=\left\{\begin{array}{ll}
Q_{i}(t), & Q_{i}(t)<Q_{i, \max } \\
Q_{i, \max }, & Q_{i}(t) \geq Q_{i, \max }
\end{array}, i=1, \ldots, m\right.
$$

where $\tilde{Q}(t)=\left[\tilde{Q}_{1}(t), \ldots, \tilde{Q}_{m}(t)\right]^{T}$ is the practical input; and $Q_{\max }=\left[Q_{1, \max }, \ldots, Q_{m, \max }\right]^{T}$ is the capacity of input links within one cycle.

Remark 4. The steady-state value $x_{e}$ of the network can be derived from the network capacity of the NMFD combined with appropriate traffic assignment, i.e., the network capacity assigned to local network links, and from (22), the steady-state input $Q^{e}$ with the given $x_{e}$ satisfies $B Q^{e}=\left(E_{n}-\bar{A}^{a}\right) L^{-1} X_{e}$.

\section{Signal Control in the Network}

The network inputs $\tilde{Q}_{i}(t), i \in V^{S}$ generated from (23) are controlled by the signal lights on boundaries of the network, 
which should ensure that the number $\tilde{Q}_{i}(t)$ of vehicles can enter the network in one cycle, i.e., the input link green-time $g_{i j}(t), i \in V^{S}$ from link $i$ to downstream link $j$ in one cycle should satisfy $\sum_{j \in V_{i}^{D}} s_{i j} g_{i j}(t) \geq \tilde{Q}_{i}(t)$, where $s_{i j}>0$ is the saturation flow of vehicles traveling from link $i$ to downstream link $j$.

For the discharging proportions $\bar{\eta}^{a}=\left[\bar{\eta}_{1}^{a}, \ldots, \bar{\eta}_{n}^{a}\right]^{T}>>0$, which can be determined by Proposition 1 and fine-tuned by value $a$ to conform with the current saturation levels in network links, from the definition of the discharging proportion in (1), the relation between $\bar{\eta}_{i}^{a}$ and the link green-time $g_{i j}(t), j \in V_{i}^{D}$ is given by:

$$
\bar{\eta}_{i}^{a}=\frac{\sum_{j \in V_{i}^{D}} s_{i j} g_{i j}(t)}{N_{i}(t)}=\frac{s_{i} g_{i}(t)}{N_{i}(t)},
$$

where $N_{i}(t)$ is the number of vehicles in link $i$ at the current time; $g_{i}(t)$ is the total green-time of link $i$ obtained in one cycle, and $g_{i j}(t)=\beta_{i j} g_{i}(t)$, where $\beta_{i j} \geq 0$ is the distributing proportion of $g_{i}(t)$ from link $i$ to link $j$ in one cycle, and satisfies: $\sum_{j \in V_{i}^{D}} \beta_{i j}=1$; and $s_{i} \triangleq \sum_{j \in V_{i}^{D}} s_{i j} \beta_{i j}$ is called the cycle average saturation flow of link $i$; Specifically, if all the flows from link $i$ to downstream links have right of way at the same time, $s_{i}$ and $g_{i}(t)$ are respectively the saturation flow and the phase green-time of link $i$. Then, the distribution of the link green-time $g_{i}(t)$ to downstream links $j \in V_{i}^{D}$ in one cycle can be determined by:

$$
\left\{\begin{array}{l}
g_{i j}(t)=\beta_{i j} g_{i}(t)=\frac{\beta_{i j} \bar{\eta}_{i}^{a} N_{i}(t)}{s_{i}}, \\
s_{i}=\sum_{j \in V_{i}^{D}} s_{i j} \beta_{i j}, \\
\beta_{i j} \triangleq \frac{\alpha_{i j} / s_{i j}}{\sum_{j_{l} \in E_{i}^{D}} \alpha_{i j_{l}} / s_{i j_{l}}},
\end{array}\right.
$$

where $\alpha_{i j} / s_{i j}$ similar to the flow ratio, indicates the load of the corresponding flow direction.

Remark 5. $\bar{\eta}^{a}=\left[\bar{\eta}_{1}^{a}, \ldots, \bar{\eta}_{n}^{a}\right]^{T}$, discharging proportions of network internal links, is solved by using results in Proposition 1, which would coincide with the current saturation levels in links, e.g., if traffic flows in links are in oversaturated state, we can properly choose the value $a$ approaching one such that for some link $i, \bar{\eta}_{i}^{a}<1$. Though, $\bar{\eta}^{a}=\left[\bar{\eta}_{1}^{a}, \ldots, \bar{\eta}_{n}^{a}\right]^{T}$ is fixed within some time interval, coinciding with the corresponding traffic states in the network, the signal control plans for junctions in the network are time-varying from (25), which are traffic-responsive to dynamic traffic flows. Explicitly, it is pointed out that the input control law of the network in (23) is of centralized type, the running of which depends on state values in all internal links, and differently the signal control for junctions of the network in (25) is of distributed type, the running of which depends on state values in local links and then is robust to detector failures.

The link green-time generated by (25) may not satisfy some constraints in practical applications. Then, the local optimization problem in each of junctions is established and then solved as follows: assume that there are $q>1$ signal phases in some junction $j \in J$, each of which contains $l_{i}, i=1, \ldots, q$ non-conflicting traffic flows, then the following optimization problem is solved each cycle, for practical signal control plans,

$$
\begin{aligned}
\min & \frac{1}{2} \sum_{i=1}^{q} \sum_{k=1}^{l_{i}}\left[G_{j}^{i}(t)-g_{j k}^{i}(t)\right]^{2} \\
G_{j i}^{\min } & \leq G_{j}^{i}(t) \leq G_{j i}^{\max }, i=1, \ldots, q \\
\sum_{i=1}^{q} G_{j}^{i}(t) & =C-L_{j}
\end{aligned}
$$

where $G_{j}^{i}(t)$ is the green-time of phase $i$ practically applied at the next cycle; $G_{j i}^{\min }$ and $G_{j i}^{\max }$ are respectively the shortest and longest green-time of phase $i$; $C$ is the common cycle of the network; $L_{j}$ is the total lost time in one cycle; and $g_{j k}^{i}(t), i=1, \ldots, q ; k=1, \ldots, l_{i}$ are the green-times generated by (25).

The quadratic optimization problem in (26) is easily solved by existing methods, which will be executed in each of junctions and indicates that the practical signal plans for each junction, as closely as possible, approach the ones generated by (25). In conclusion, the critical points and control structure for the network in our proposed approaches are summarized in Figure 3.

\section{SIMULATION INVESTIGATIONS}

In this Section, the proposed approaches are applied to a network with the topology in Yizhuang District of Beijing, and compared with the fixed-time signal control (FSC) approach conducted in Paramics simulation environment. 


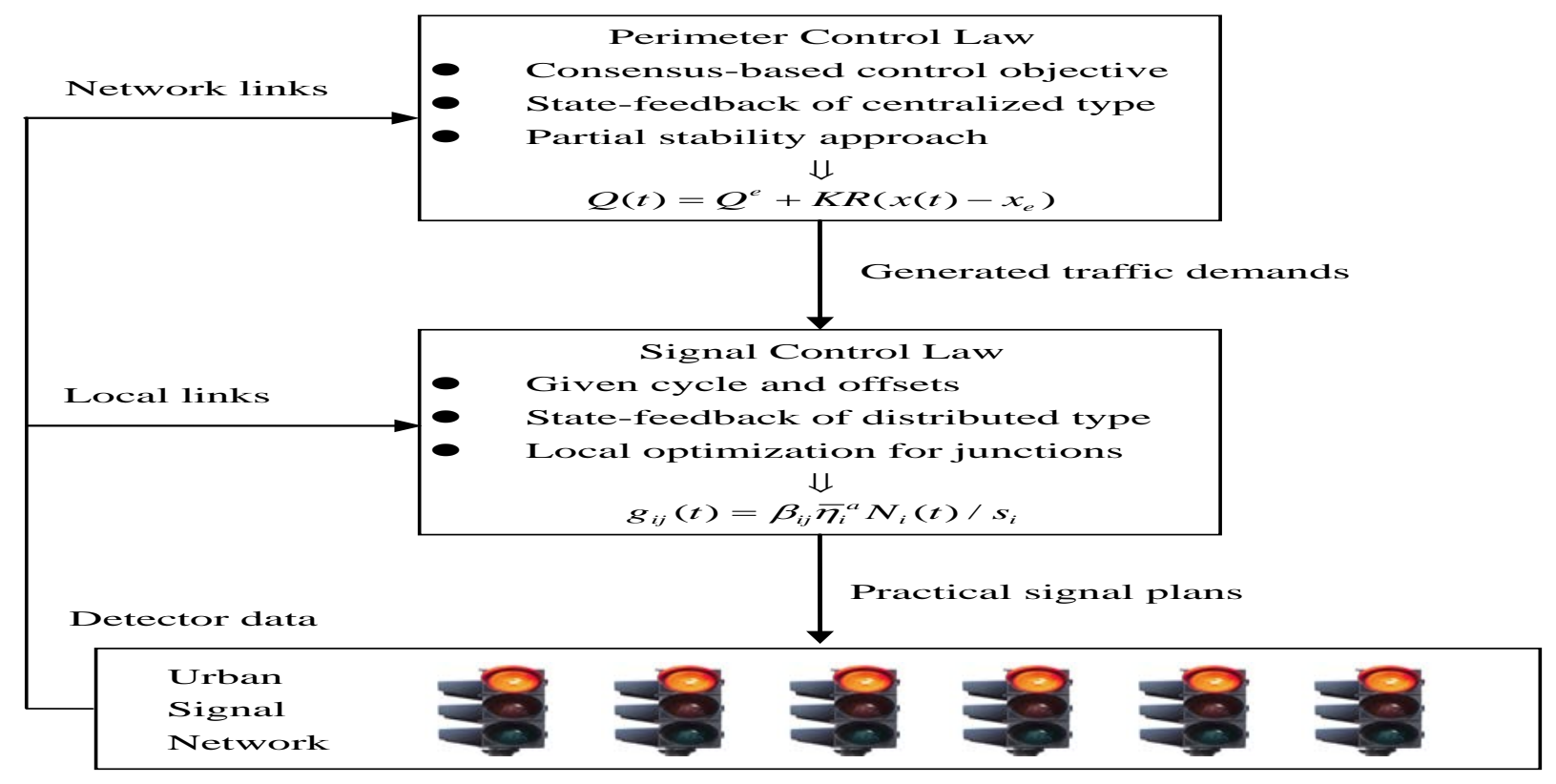

Figure 3. Control structure of the network.

The topology of simulation network is illustrated in Figure 4, which consists of 16 signalized intersections, i.e., $|J|=16$, 48 internal links, i.e., $\left|V^{I}\right|=48$, and 6 input links and output links respectively, i.e., $\left|V^{S}\right|=\left|V^{D}\right|=6$.

In this specific application, the value $a$ in Proposition 1 is fine-turned to be chosen as $a=0.94$, and the transformation matrix $P \in \mathbb{R}^{48 \times 48}$ in (9) is simply chosen as:

$$
P=\left[\begin{array}{ccccc}
1 & -1 & 0 & \cdots & 0 \\
0 & 1 & -1 & \cdots & 0 \\
\vdots & \vdots & \ddots & \cdots & 0 \\
0 & 0 & \cdots & 1 & -1 \\
1 & 1 & \cdots & 1 & 1
\end{array}\right]
$$

For the determination of $Q(t)=Q^{e}+K R\left(x(t)-x_{e}\right)$, the given matrix $R \in \mathbb{R}^{47 \times 48}$ is chosen as $R=P_{1}$, where $P_{1}$ is the first 47 rows of matrix $P$, obviously $R 1_{48}=0$ from the construction of the matrix $P$, the feedback-gain matrix $K \in \mathbb{R}^{6 \times 47}$ can be numerically solved by using the Matlab LMI Toolbox, and the expected values of $x_{e}$ and $Q_{e}$ can be determined referring to statements in Remark 4.

It is derived from Proposition 3 and discussions followed, that the consensus decision function $X^{*}(t) \rightarrow 0$ for the difference system with $X \triangleq x-x_{e}$ and $\hat{Q} \triangleq Q-Q^{e}$, then we have $\lim _{t \rightarrow \infty}\left[x(t)-x_{e}\right]=0$ and $\lim _{t \rightarrow \infty}\left[Q(t)-Q^{e}\right]=0$, which can be validated in Figure 5.

The performance evaluation of the proposed approaches is conducted in Paramics simulation software, and the simulation period is given by $2 \mathrm{~h}$ with 100 s sampled period. The given OD demands of the network is presented in Table I, and simulation test results in Table II indicate that the proposed approach realizes improvements of all performance indices of the simulation network compared with the FSC approach, where the FSC plan of the network is determined with the simulation-based data and observation-based fine-tuned process. Furthermore, the comparisons of average delay and travel time are respectively illustrated in Figure 6 and 7, where the performance of most of local links using the proposed approach is better than that using the FSC approach, especially in local congested links, and because of the consensus-based control objective of the proposed approach, the evolution of the performance indices is smooth compared to the FSC approach.

Let $T_{i j}$ be the travel time from Zone $i$ to Zone $j$, and then $T_{i}=[1 / 6] \sum_{j \neq i} T_{i j}$ is the average travel time of Zone $i$, which reflects the congestion levels in some routes, and is evaluated using the two approaches in Table III, showing the advantage of our methods.

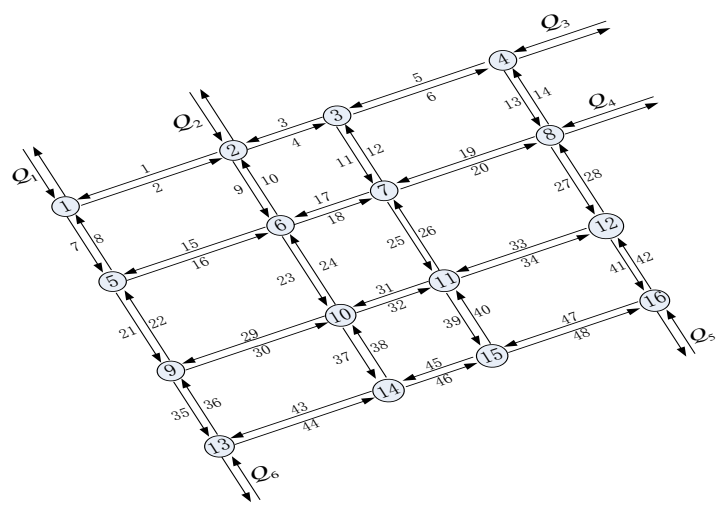

Figure 4. Topology of the simulation network. 


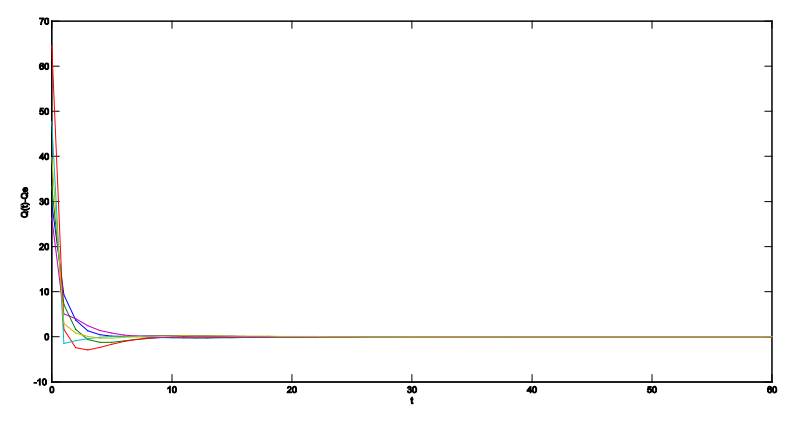

(a)Evolution of the difference $Q(t)-Q^{e}$

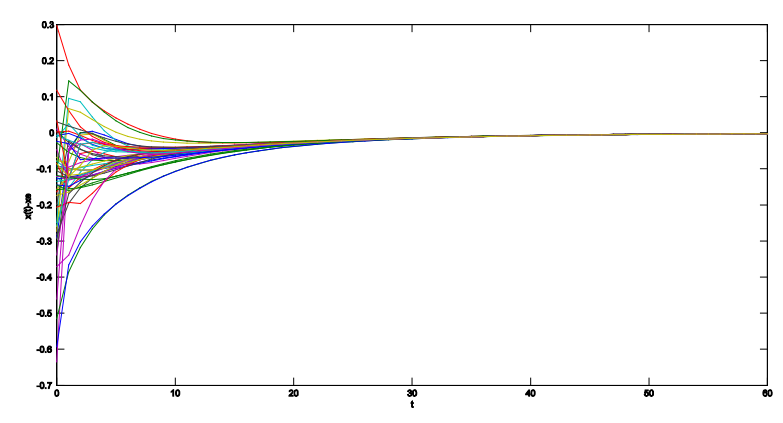

(b)Evolution of the difference $x(t)-x_{e}$

Figure 5. Evolution of the inputs and states of the network.

\section{TABLE I. TABLE OD DEMANDS OF THE NETWORK}

\begin{tabular}{|lllllllll|} 
& Zone 1 & Zone 2 & Zone 3 & Zone 4 & Zone 5 & Zone 6 & Total \\
\hline Zone 1 & & 120 & 250 & 150 & 300 & 260 & 1080 \\
\hline Zone 2 & 180 & & 100 & 200 & 300 & 300 & 1080 \\
\hline Zone 3 & 100 & 50 & & 80 & 190 & 120 & 540 \\
\hline Zone 4 & 200 & 178 & 130 & & 200 & 300 & 1008 \\
\hline Zone 5 & 220 & 210 & 178 & 100 & & 120 & 828 \\
\hline Zone 6 & 270 & 200 & 200 & 260 & 150 & & 1080 \\
\hline Total & 970 & 758 & 858 & 790 & 1140 & 1100 & 5616 \\
\hline
\end{tabular}

TABLE II. TABLE COMPARISON OF THE NETWORK PERFORMANCE

\begin{tabular}{ccc}
\hline Performance & FSC & Improved \\
\hline Average Delay (s) & 29.45 & 21.28 \\
Change Percentage & & $-27.75 \%$ \\
\hline Input Link Delay (s) & 65.53 & 59.46 \\
Change Percentage & & $-9.26 \%$ \\
\hline Average Stopped Delay (s) & 3.14 & 2.62 \\
Change Percentage & & $-16.57 \%$ \\
\hline
\end{tabular}

TABLE III. TABLE COMPARISON OF TRAVEL TIME OF ZONES

\begin{tabular}{ccccccc}
\hline & Zone 1 & Zone 2 & Zone 3 & Zone 4 & Zone 5 & Zone 6 \\
\hline FSC [s] & 460 & 449 & 573 & 428 & 557 & 462 \\
Improve & 341 & 342 & 374 & 313 & 423 & 489 \\
$\mathrm{~d}$ [s] & & & & & & \\
Change & -25.87 & -23.83 & -34.73 & -26.87 & -24.06 & +5.84 \\
& $\%$ & $\%$ & $\%$ & $\%$ & $\%$ & $\%$ \\
\hline
\end{tabular}

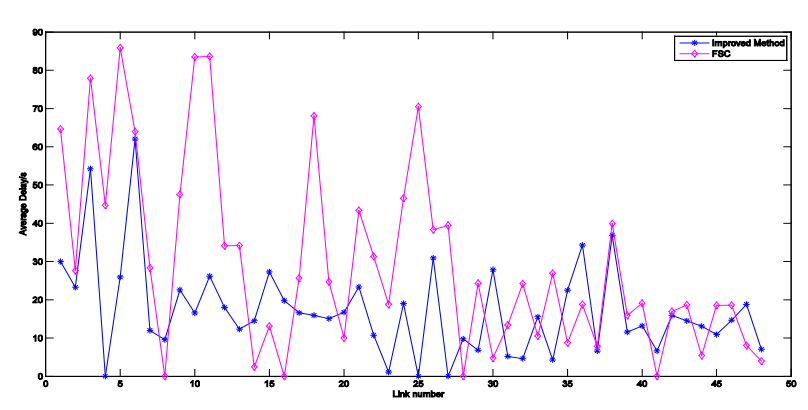

Figure 6. Comparison of average delay in links.

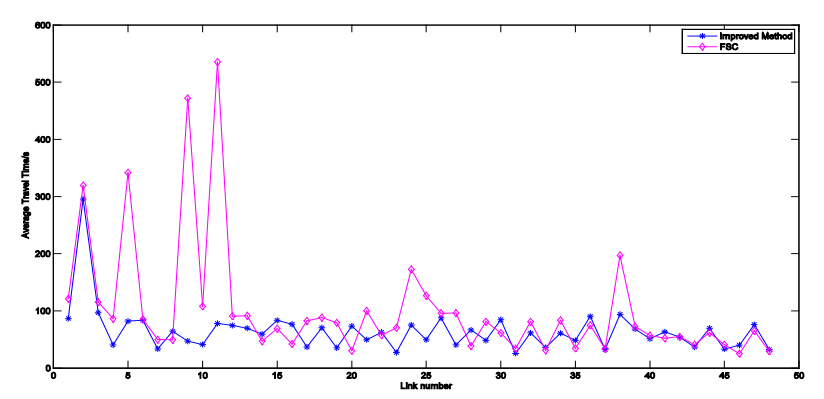

Figure 7. Comparison of average travel time in links.

\section{CONCLUSIONS}

This paper develops design approaches for the perimeter feedback control of the network, which realizes the consensus of the network link occupancies and then the balanced distribution of the network flows, and more importantly guarantees the maximum network capacity. Also, a simple feedback signal control approach of the network is provided combined with the network perimeter control law.

The proposed perimeter control law would be applied to more practical networks and compared with other approaches. Moreover, developments of the theoretical methods for the distributed extension of the perimeter control approach would be also our focus in the future.

\section{ACKNOWLEDGMENT}

Research supported by NSFC (Grant No. 51308005 and 61374191); Scientific Research Project of Beijing Education Committee (Grant No. PXM2016-014212-000036, PXM2016-014212-000030). 


\section{REFERENCES}

[1] M. Papageorgiou, Applications of Automatic Control Concepts to Traffic Flow Modeling and Control. Berlin: Springer-Verlag, 1983.

[2] M. Papageorgiou, C. Diakaki, V. Dinopoulou, A. Kotsialos, and Y.B. Wang, "Review of road traffic control strategies," Proceedings of the IEEE, vol. 91, pp. 2043-2067, December 2003.

[3] H. K. Lo, "A cell-based traffic control formulation: strategies and benefits of dynamic timing plans," Transportation Science, vol. 35, pp. 148-164, May 2001.

[4] H. K. Lo, E. Chang, and Y.C. Chan, "Dynamic network traffic control,” Transportation Research Part A: Policy and Practice, vol. 35, pp. 721-744, September 2001.

[5] S. Lin, B.De. Schutter, Y.G. Xi, and H. Hellendoorn, "Fast model predictive control for urban road networks via MILP," IEEE Transactions on Intelligent Transportation Systems, vol. 12, pp. 846-856, September 2011.

[6] K. Aboudolas, M. Papageorgiou, A. Kouvelas, and E. Kosmatopoulos, "A rolling-horizon quadratic-programming approach to the signal control problem in large-scale congested urban road networks," Transportation Research Part C: Emerging Technologies, vol. 18, pp. 680-694, October 2010.

[7] C. Diakaki, M. Papageorgiou, and K. Aboudolas, "A multivariable regulator approach to traffic-responsive network-wide signal control," Control Engineering Practice, vol. 10, pp. 183-195, February 2002.

[8] K. Aboudolas, M. Papageorgiou, and E. Kosmatopoulos, "Store-and-forward based methods for the signal control problem in large-scale congested urban road networks," Transportation Research Part C: Emerging Technologies, vol. 17, pp. 163-174, April 2009.

[9] I. Ioslovich, J. Haddad, P.O. Gutman, and D. Mahalel, "Optimal traffic control synthesis for an isolated intersection," Control Engineering Practice, vol. 19, pp. 900-911, August 2011.

[10] B.De. Schutter, "Optimizing acyclic traffic signal switching sequences through an extended linear complementarity problem formulation," European Journal of Operational Research, vol. 139, pp. 400-415, June 2002.

[11] J. Haddad, B.De. Schutter, D. Mahalel, I. Ioslovich, and P.O. Gutman, "Optimal steady-state control for isolated traffic intersections," IEEE Transactions on Automatic Control, vol. 55, pp. 2612-2617, November 2010.

[12] Z.H. He, Y.Z. Chen, J.J. Shi, X.G. Han, and X. Wu, "Steady-state control for signalized intersections modeled as switched server system," in Proceedings of the 2013 American Control Conference. New York: IEEE, 2013, pp. 842-847.

[13] L. Wang, Z.H. He, and C. Zhang, "Stability of switched server systems with constraints on service-time and capacity of buffers," Mathematical Problems in Engineering, vol. 2015, pp. 1-10, 2015.

[14] J. Haddad, D. Mahalel, I. Ioslovich, and P.O. Gutman, "Constrained optimal steady-state control for isolated traffic intersections," Control Theory and Technology, vol. 12, pp. 84-94, February 2014.

[15] Z.H. He, Y.Z. Chen, J.J. Shi, X. Wu, and J.Z. Guan, “Consensus based approach to the signal control of urban traffic networks," Procedia Social and Behavioral Sciences, vol. 96, pp. 2511-2522, November 2013.
[16] L. Wang, D. Li, X.M. Liu, and Z.X. Li, "Regional traffic state consensus optimization based on computational experiments," in Proceedings of the 16th International IEEE Annual Conference on Intelligent Transportation Systems. New York: IEEE, 2013, pp. 1547-1552.

[17] L. Wang, D. Li, Z.H. He, and X.H. Ma, "Urban traffic network control based on cluster consensus of multi-agent systems," Control Theory \& Applications, vol. 31, pp. 1448-1456, November 2014. (in chinese)

[18] Z.H. He, L. Wang, D. Li, L.Y. Zhang, "Steady-state signal control for urban traffic networks," in Proceedings of 18th IEEE International Conference on Intelligent Transportation Systems. New York: IEEE, 2015, pp. 463-470.

[19] K. Jang, H. Kim, and I.G. Jang, "Traffic signal optimization for oversaturated urban networks: queue growth equalization,” IEEE Transactions on Intelligent Transportation Systems, vol. 16, pp. 2121-2128, August 2015.

[20] C.F. Daganzo, N. Geroliminis, "An analytical approximation for the macroscopic fundamental diagram of urban traffic," Transportation Research Part B: Methodological, vol. 42, pp. 771-781, November 2008.

[21] N. Geroliminis, C.F. Daganzo, "Existence of urban-scale macroscopic fundamental diagrams: some experimental findings," Transportation Research Part B: Methodological, vol. 42, pp. 759-770, November 2008.

[22] N. Geroliminis, J. Sun, "Properties of a well-defined macroscopic fundamental diagram for urban traffic,” Transportation Research Part B: Methodological, vol. 45, pp. 605-617, March 2011.

[23] M. Keyvan-Ekbatani, A. Kouvelas, I. Papamichail, and M. Papageorgiou, "Exploiting the fundamental diagram of urban networks for feedback-based gating," Transportation Research Part B: Methodological, vol. 46, pp. 1393-1403, December 2012.

[24] M. Keyvan-Ekbatani, M. Yildirimoglu, N. Geroliminis, and M. Papageorgiou, "Multiple concentric gating traffic control in large-scale urban networks," IEEE Transactions on Intelligent Transportation Systems, vol. 16, pp. 2141-2154, August 2015.

[25] K. Aboudolas, N. Geroliminis, "Perimeter and boundary flow control in multi-reservoir heterogeneous networks," Transportation Research Part B: Methodological, vol. 55, pp. 265-281, September 2013.

[26] N. Geroliminis, Jack Haddad, and M. Ramezani, “Optimal perimeter control for two urban regions with macroscopic fundamental diagrams: a model predictive approach,” IEEE Transactions on Intelligent Transportation Systems, vol. 14, pp. 348-359, March 2013.

[27] K. Ampountolas, N. Zheng, and N. Geroliminis, "Robust control of bi-modal multi-region urban networks: an LMI optimisation approach," in Proceedings of 17th IEEE International Conference on Intelligent Transportation Systems. New York: IEEE, 2014, pp. 489-494.

[28] D.C. Gazis, "Optimum control of a system of oversaturated intersections,” Operations Research, vol. 12, pp. 815-831, 1964.

[29] A. Berman, and R.J. Plemmons, Nonnegative Matrices in the Mathematical Sciences. Philadelphia: SIAM, 1994.

[30] S. Boyd, L.E. Ghaoui, E. Feron, and V. Balakrishnan, Linear Matrix Inequalities in System and Control Theory. Philadelphia: SIAM, 1994. 